\title{
Testing the theory: demonstration projects and the validation of integrated design protocols for advanced energy retrofits
}

\author{
F. Trubiano, K. Albee \& M. Brennan \\ University of Pennsylvania, USA
}

\begin{abstract}
This paper details an initial set of observations resulting from the launch of five building retrofit demonstration projects currently underway for deploying the Integrated Design (ID) Advanced Energy Retrofit (AER) Roadmap; a marketoriented product developed by the authors of this paper for accelerating the pace of advanced energy retrofits in the United States (US). This project is one of many dedicated to improving the energy profile of existing buildings at the Consortium for Building Energy Innovation (CBEI) - formerly the Energy Efficient Buildings $\mathrm{Hub}$ - a research initiative sponsored by the Building Technologies Office of the US Department of Energy.

Achieving significant energy reductions in the renovation of small to mediumsized commercial building remains an elusive goal, albeit an important one. To this end, the three-part document suite that is the ID AER Roadmap was conceived to offer all participants involved in the actual design, construction and operations of a retrofit project the management based tools to create a collaborative, replicable process that assures greater energy reductions following the retrofit of a building's various material and engineering systems. This paper discusses a number of important initial results from the testing and verification of the roadmap's seven ID process protocols. Discussed in some detail is the particular method by which each of the five project teams has employed the various components of the ID AER Roadmap, and where instructive, client profiles and building type differences will be identified to the extent that they impact the execution of the ID protocols of the AER Roadmap.

Keywords: integrated design, advanced energy retrofits, demonstration projects, energy efficiency, design roadmaps, commercial buildings.
\end{abstract}




\section{Introduction}

This paper details the testing and verification of an Integrated Design (ID) Advanced Energy Retrofit (AER) Roadmap across five demonstration projects that are currently underway at the Consortium for Building Energy Innovation (CBEI). As part of this work, a set of roadmap documents for organizing the actual design and construction process of an AER have been developed to integrate and coordinate the full range of architecture, engineering, and construction (AEC) services required in the execution of a retrofit. The ID AER Roadmap is comprised of a three-tiered document suite that outlines the process by which service providers are encouraged to implement integrated design strategies for increasing the completion of advanced energy retrofits; the goal of which is to transform the energy efficiency market of existing small to medium-sized commercial buildings. The particulars of this Roadmap are the subject of the demonstration projects, and the CBEI has sponsored their facilitation.

The larger project to design the ID AER Roadmap was begun in 2011, the details of which are published in the paper, Advanced Energy Retrofit-Designing Integrated Design Roadmaps (Trubiano et al. [1]), while Deployment and Testing of Integrated Design Roadmaps for Advanced Energy Retrofits (Albee et al. [2]) details the various roadmap documents used in organizing the demonstration projects.

This particular paper details the CBEI initiative begun in 2013 to test, verify and where possible, enhance the design and conceptual structure of the $I D A E R$ Roadmap by way of the feedback from five different demonstration projects; each of which were sourced from owner groups interested in completing an advanced energy retrofit. Alongside the testing of technology solutions by other researchers at the CBEI, an important goal of this initiative was to use real project constraints to challenge the process-based ID protocols designed for the roadmap and of use to the AER industry. As such, this paper reviews the implementation and initial results from testing our ID process protocols on the demonstration projects; as well as the impact this has had on the structure and organization of the roadmap.

\section{ID AER Roadmap concepts}

As outlined in Advanced Energy Retrofit - Designing Integrated Design Roadmaps (Trubiano et al. [1]), a total of seven Integrated Design (ID) protocols and four distinct retrofit scales serve as the conceptual foundations of the $I D A E R$ Roadmap. These process-based ID protocols are to be implemented within all retrofit projects, regardless of scale. They include (1) an all team project mission statement; (2) participation in integrated design requests for proposals; (3) participation in process oriented collaborative meetings; (4) commitment to 'energy free' design solutions; (5) commitment to whole building systems design; (6) commitment to predictive modeling; and (7) commitment to measurement and verification $(\mathrm{M}+\mathrm{V})$. 
Furthermore, the identification of four retrofit scales was needed in order to acknowledge how, contrary to the construction of new buildings, the retrofit of existing buildings typically occurs at various scopes of engagement, as no two retrofits are entirely alike. The four scales identified for the sector of advanced energy retrofits are lite, partial, substantial, and comprehensive, with each scale having a corresponding Project Team Guide in order to organize the seven protocols into actionable checklists and corresponding guidelines. In this way, building owners and their project teams are guided step by step in the implementation of the ID protocols.

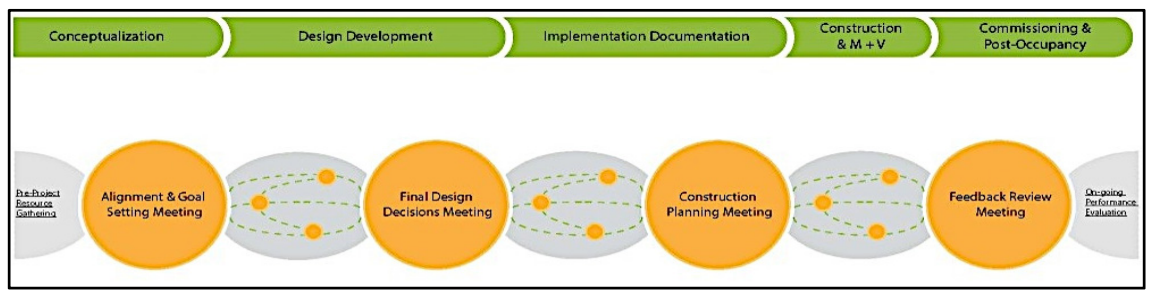

Figure 1: ID AER Roadmap ID process diagram - partial retrofits.

The Project Team Guide used by each of the demonstration project teams details five phases of work common to any retrofit project (conceptualization, design development, implementation documentation, construction and $\mathrm{M}+\mathrm{V}$ and commissioning and post occupancy). Each phase requires the collaboration of all professionals on the team, with the depth of their individual roles varying from task to task. In addition, each phase is defined by two distinct stages - a resource gathering (RG) stage and a collaborative meeting (CM) stage (see Figure 1). A detailed description of the various documents used in each of the RG and CM stages can be found in the paper, Deploying and Testing Integrated Design Roadmaps for Advanced Energy Retrofits, by the same authors. More generally, $\mathrm{RG}$ stages involve the collection of all forms of data and information required for the retrofit process, while CM stages involve face-to-face meetings with all of members of the project team.

What follows is a description of the various project types chosen by the CBEI to test the numerous process theories that organize both the roadmap and its larger document suite. Lastly, by engaging five different building types and owner groups, significant observations have been made on both the capacities and limitations of the ID AER Roadmap, as originally designed.

\section{Demonstration projects}

\subsection{Building A}

The first demonstration project, which this paper identifies as Building A, is located within a 1,200-acre campus that is the US Navy Yard in Philadelphia, PA. It is approximately $75,000 \mathrm{ft}^{2}$ in size, with a basement and three floors above ground (see Figure 2). First built in 1911 for use by the military as barracks, the 
building is a load bearing brick masonry structure with heavy wood timber floor framing. It recently underwent a major refurbishment in 1999 to accommodate its new function as a commercial building. Thereafter, additional efforts were undertaken to create separate tenant spaces, albeit at that time existing building systems were not fully upgraded to address the new layout. (CBEI [3])

The owner of Building A is considered an informed client; being a non-profit public entity with experience in the completion of AER projects and the proprietor of a large portfolio of real estate holdings. Moreover, an experienced commercial manager has been involved since beginning of the project, serving in the role of project manager. Once the ID AER project initiated, the owner brought on building industry professionals including consultants in mechanical systems, electrical systems, and building automation and control systems; albeit most of them had but a limited amount of previous experience with ID and Integrated Project Delivery (IPD).

The owner of Building A was motivated to complete the AER project because of increasing energy costs, tenant comfort issues, and impending equipment replacement due to projected unit end-of-life scenarios. With assistance from $\mathrm{CBEI}$ researchers, an $\mathrm{M}+\mathrm{V}$ system was installed in 2011 to establish pre-retrofit energy baselines and to facilitate predictive modeling. Through extensive site assessment work, CBEI researchers identified several energy efficient measures (EEMs) to implement as part of the ID AER project. These included the use of enhanced building automation and controls, the replacement of a condenser unit, and a variety of envelope measures focused on reducing air infiltration.

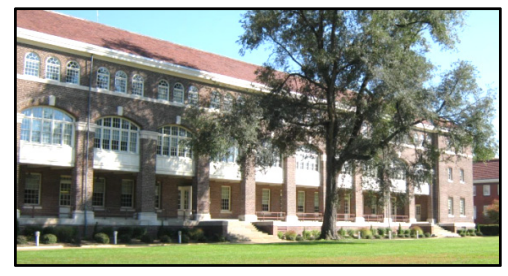

Figure 2: Building A exterior.

\subsection{Building B}

The second demonstration project, Building B, is approximately $45,000 \mathrm{ft}^{2}$ in size; it extends over a single story and has only a partial, unoccupied basement (see Figure 3). Initially built in the early 1960s, an addition was added to the building in 1976. It serves as a medical clinic and is part of a large inner city municipal public health system. The building includes two courtyard spaces enclosed by skylights built of single-paned windows. And only most recently it was fitted with a building automation system.

Building B is represented by a complicated owner group which is being administered by municipal representatives from different departments, each of whom have varying goals and motivations for the retrofit project. On the one hand, a motivated sustainability manager is the main project funder who serves in the 
role of project manager. He is driven by the promise of energy cost savings as well as by the potential for the project to identify a replicable process for retrofitting future municipal projects. On the other hand, the owner group consists of health system representatives, who are less informed about the potential for gains from undertaking such an activity, and this includes the building's facility manager. Yet, of the five demonstration projects underway, Building B has the most informed professional project team members, including an architect with extensive ID/IPD experience; an energy management consultant; and a knowledgeable engineer.

As part of the original site assessment work, CBEI researchers identified several systems based engineering issues within the building needing to be addressed. This resulted in a list of preliminary EEMs including a lighting retrofit, the use of enhanced building automation and controls, and several possible envelope measures including the addition of roof insulation, the prevention of air infiltration, and the replacement of single-pane windows.

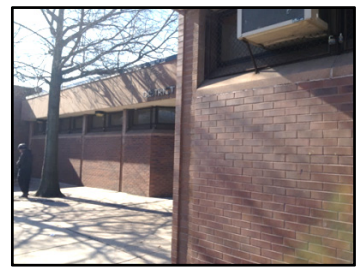

Figure 3: Building B exterior.

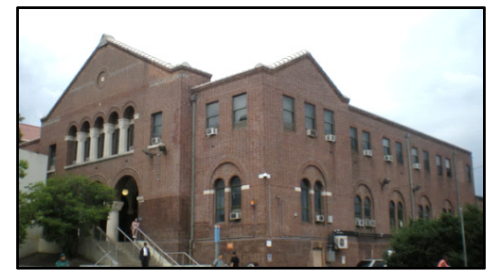

Figure 4: Building C exterior.

\subsection{Building $\mathrm{C}$}

The third demonstration project, termed Building $\mathrm{C}$, involves the retrofit of a large turn of the century transportation hub. The large span two-story brick masonry building measures approximately $50,000 \mathrm{ft}^{2}$ (see Figure 4) and was initially built in 1907. It was last renovated in 1986 when it underwent an extensive systems based retrofit. The building is still used as a major regional rail and bus hub with a high volume of users (more than 30,000 customers daily). It is operated 24 hours a day, seven days a week.

Building $\mathrm{C}$ also has a fairly complicated owner group structure, whose decisions-makers represent various sectors of the regional transportation agency. members of the Finance Department are the project's main sponsors, while a senior engineer serves in the role of project manager. Members of the Finance Department manage the sustainability goals for the whole organization and are highly motivated to complete the project, seeking to achieve greater levels of cost savings. However, not all team members are as equally motivated. In addition, differently than the four other demonstration projects, this owner group plans to fund their retrofit through the use of an energy service company (ESCO) contract.

As a result of the site assessment work conducted by CBEI researchers, preliminary EEMs were identified for this project as well; including a lighting retrofit, the replacement of existing boilers, the installation of building controls, and envelope measures focused on enhancing the building's entranceways. 


\subsection{Building D}

The fourth demonstration project, Building D, is located on a 22-acre park-like campus used by a religious social services organization. It is a $54,000-\mathrm{ft}^{2}$ structure which has two primary uses. The top two floors are used as transitional residential apartments and administrative staff space, while the lower two floors are used as flexible programmable spaces that include a summer camp, an after-school program, as well as a public computer center and additional administrative offices. This signature building of load bearing stone was originally built in 1889 with additional wings completed in 1898. It is an exceptional construction of heritage status and it was most recently upgraded with new insulated aluminum windows, throughout.

The owner of Building D is a small non-profit whose representatives have little experience working on energy efficiency retrofits. However, the larger owner group consists of a committed individual from the contracts division, a facilities and operations manager, and a project manager. They have also incorporated within their team an architect familiar with ID and an HVAC contractor, committed to the group's mission. Most critically, the group is motivated to engage in an ID AER project in order to ensure energy savings for their organization, which has a very limited operating budget. In addition, they hope to replicate their successes on additional projects, given that they own several other buildings within the same campus.

The CBEI's site assessment work identified issues within the existing building that needed to be addressed and proposed corresponding preliminary EEMs for the project. These included an overhaul of the existing heating and cooling systems, building envelope work to reduce energy demand, replacement and/or upgrade of existing controls based on modified space planning, and a lighting retrofit.

\subsection{Building $\mathbf{E}$}

The fifth demonstration project, Building E, is a three-story building, approximately $18,000 \mathrm{ft}^{2}$ in size. It is a mixed-use building with three separate programs; municipal offices, a police department, and even a public library (see Figure 6). The building was constructed in 1889 as a load bearing brick masonry building and today has insulated windows and an asphalt shingle roof. In 2003,

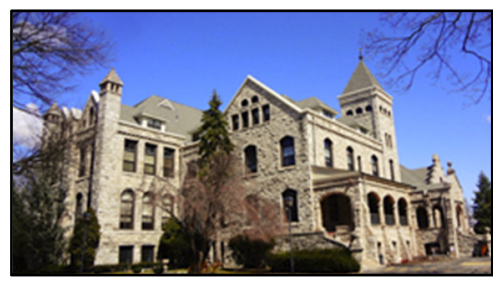

Figure 5: Building D exterior.

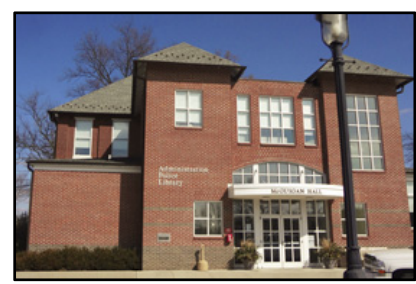

Figure 6: Building E exterior. 
the building was renovated to improve its safety systems and to update its level of code compliance.

The owner of Building $\mathrm{E}$ is a small municipality whose owner group members also have limited experience working on energy efficiency projects. Its membership consists of the current elected council president, the municipality's administration manager, as well as a representative responsible for zoning and code enforcement. The owner was motivated by a need for increased occupant comfort, and the impending end of life of an existing boiler.

Site assessment work completed by the CBEI researchers identified several possible EEMs for Building E. These included the replacement of the existing HVAC oil-fired boiler, the addition of HVAC controls, and enhancement of the building's existing automation system controls. Additionally, building envelope improvements were identified including upgrades to the existing windows, exterior walls, and attic spaces.

\section{Implementing ID protocols within demonstration projects}

At the end of 2013, a core team of researchers was identified to work on the five demonstration projects, described herein above. The team included the CBEI's demonstration project manager, his modeling and measurement team $(\mathrm{M}+\mathrm{M})$, the ID AER Roadmap project research team (paper's authors), and a professional ID facilitator acting on behalf of the CBEI. The process began with the CBEI hosting an initial collaborative meeting $(\mathrm{CM})$ stage, termed the "pre-alignment workshop." This was attended by all of the demonstration project owner groups and deemed necessary in order to launch the facilitation of these projects for the CBEI. (This is why the pre-alignment workshop is unique to these demonstration projects and is not listed in ID process diagram, as seen in Figure 1). Furthermore, the pre-alignment workshop was conceived to help all five demonstration project teams become familiar with the ID process they would be undertaking and inform them of the role of CBEI researchers within the process.

This pre-alignment workshop was facilitated by an ID expert with the goal of helping each project team recognize the important of having all of its members "aligned" with the project's intentions, procedures, and outcomes. As part of this workshop, the authors outlined the concepts within the ID AER Roadmap and the Project Team Guide documents that each team would be using throughout their retrofit journey. In addition, a high level presentation was made on the integrated process with outcomes focused on purpose, values, and goals. During the workshop, project teams participated in collaborative exercises to understand the value of the integrated process and to identify high level project-specific goals and team roles, as well as the required next steps.

This was the first opportunity to begin testing the design of the roadmap against the interactions and comments each project team put forth during the workshops. To this end, each team completed the "Scale of Retrofit Quiz" designed to help them get a better understanding of the scale and scope of their project. Additionally, a brief survey was administered to the participants at the conclusion of the workshop to gather feedback for the further development of the roadmap. 


\subsection{Conceptualization phase: pre-project resource gathering stage}

Following the pre-alignment workshop, the pre-project resource gathering (RG) stage was initiated for each of the demonstration projects. This included the use of the Project Team Guide documents by the owner/project manager and the modeling and measurement professionals (from CBEI) in order to complete the required checklist activities. These activities included the review of financial resources, the gathering of building centered information, the development of preretrofit baselines, and the contracting of professional team members. This RG stage also included a site assessment meeting held separately with each of the owner groups to review the preliminary evaluation of the building's physical infrastructure and engineering systems conducted by the CBEI researchers in order to identify the project's potential EEMs.

In addition, the core team continued to collaborate to develop plans for the $I D$ AER Roadmap's first CM stage identified as the "alignment and goal setting meeting." This CM stage was conducted with each of the demonstration project teams in separate sessions in order to assist them in better understanding the ID process, in developing an all team mission statement, and in aligning around project goals specifically tailored to their retrofit. In advance of the CM stage, a series of agenda setting conference calls were held with all of the owner groups to prepare for the alignment and goal setting meeting. Moreover, this conference call helped all participants understand the expectations and necessary preparations required to maximize the effectiveness of the $\mathrm{CM}$ stage. An essential part of which was the need for the entire project team (owner group and AEC consultants) to be present and fully engaged to take important decisions attendant to a mission statement.

\subsection{Conceptualization phase: alignment and goal setting collaborative meeting stage}

In early 2014, the core team conducted the alignment and goal setting meeting with all project team members in attendance to discuss and organize the major framework for their AER project (see Figure 7). To accomplish this task, project team members, with the aid of CBEI researchers and facilitators, outlined an agreed to Mission Statement, which is the first of the seven ID protocols necessary to the ID AER Roadmap. Thereafter, the same team members agreed on the

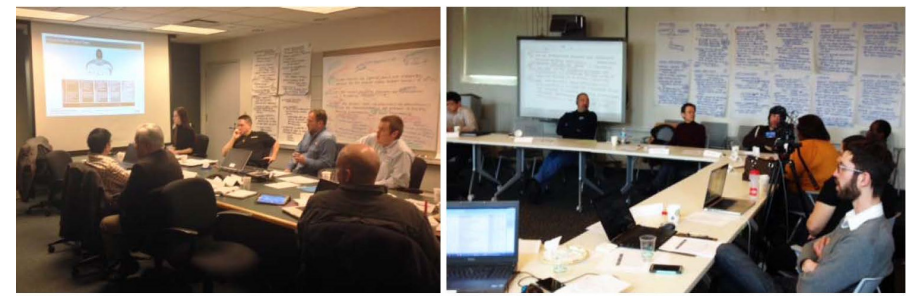

Figure 7: Alignment and goal setting CM stage. 
project's goals and constraints, as well as reviewed the core ID protocols labeled four to seven in this paper (including energy free design solutions, whole building systems, predictive modeling, and measurement + verification). In addition, the project team reached alignment around the following priorities; the project's human centered and energy performance goals; its cost priorities; and its scope, budget, and schedule.

\subsection{Design development phase: resource gathering stage}

Following the alignment and goal setting meeting, the demonstration project teams initiated work on the design development phase, which included a series of subteam meetings to review and finalize the project's scope. Having established that most of the demonstration projects are partial scale retrofits, three additional meetings were set to encourage the collaboration of project team members in selecting the final project EEMs. Sub-team meeting 1 is intended to focus on the review energy free design solutions proposed by the team's architect; whole building systems design solutions developed by the project's engineer, and the $\mathrm{M}+\mathrm{V}$ plan developed by the AEC and $\mathrm{M}+\mathrm{M}$ consultants. Sub-team meeting 2 focuses on the review of the predictive modeling analysis of potential energy savings of the selected EEMs in order to make adjustments to the project scope based on the results. Lastly, sub-team meeting 3 is intended to review the final predictive modeling results to determine the final project scope of EEMs.

Following the alignment and goal setting CM stage, some of the projects required the gathering of additional $\mathrm{M}+\mathrm{V}$ site assessment information within the design development phase because analysis work done in the conceptualization phase was insufficient for the process. As a result, prior to sub-team meeting 1, CBEI investigators completed an additional site visit to develop a better understanding of energy loads within Buildings B, C, and D in order to be able to calibrate the predictive energy model. Part of this process included the installation of $\mathrm{M}+\mathrm{V}$ equipment to gather actual pre-retrofit building energy usage data. The roadmap documents identify this work should be completed in the conceptualization phase of the project. However, due to programming uncertainties within the CBEI/EEB hub, this did not take place during the demonstration projects herein described.

\section{Impact of demonstration projects on the ID AER Roadmap's development}

Several key observations can be made and lessons learned from the testing of the ID AER Roadmap on five demonstration projects, albeit all projects have completed but $20 \%$ of the process. Interactions with owner groups and AEC consultants, direct observations during CM stages, and survey-based feedback are some of the sources for the information the research team has gathered. As a result, modifications to the ID AER Roadmap are presently underway in order to create a market product that can truly be used independently of CBEI facilitation. 


\subsection{Feedback from the pre-alignment workshop}

Feedback collected during the pre-alignment workshop confirms that many participants believed this initial all group meeting was valuable for both themselves and their projects. Participants reported they gained a deeper understanding of the ID process and its associated benefits when members of the team work collaboratively in achieving greater energy savings. The owner groups all gave positive feedback regarding their participation, and they were optimistic they would be able to meet their goals throughout the project's development. Albeit, this workshop was not originally planned as part of the ID AER Roadmap, based on feedback from participants and observations made by our research group, consideration is being given to the possible modification of the ID process diagram to include a pre-alignment CM stage in advance of the conceptualization phase.

Furthermore, it was noted that access to the following information would have been beneficial to the participants; such as the value, constraints and opportunities of phasing the project rather than completing the retrofit in one contract; an expansion of the definition of 'owner' in order to acknowledge a wider variety of ownership organizations; and finally, a greater degree of clarity within the checklist documents to identify required submittals and/or deliverables for each activity.

\subsection{Alignment and goal setting collaborative meeting stage feedback}

The demonstration project participants reconfirmed the value of the initial prealignment workshop while participating in the alignment and goal setting meeting. Clearly, those who had not been able to attend the pre-alignment workshop were vastly more hesitant in their participation during the alignment and goal setting Meeting and some were even skeptical of the value of using an ID process. For these participants, this meeting helped them recognize the value of participating in collaborative activities to identify shared project goals.

Another important observation from this CM stage was the fact that the amount of time needed for this meeting varied based on the amount of experience the team had with the principles of ID and with the specifics of the existing building. As such, some teams completed detailed goals for each of the seven ID protocols, while others struggled to complete this task in the time allocated. However, all teams completed their detailed project mission statement, a process highly facilitated by the professional ID facilitator.

In fact, the participation of a professional ID facilitator has given rise to a series of critical observations regarding the level of independence the roadmap assumes is possible when retrofit projects are not administered by the CBEI. Is the ID AER Roadmap robust and articulate enough to successfully result in the completion of each of the seven protocols without the aid of a skilled ID professional? To respond to this observation, it was decided the roadmap should include a written framework to guide team members in developing the project mission statement, a sample CM agenda, and examples of how goals can be set for each of the seven ID protocols. 


\section{Initial conclusions}

The first important conclusion identified by this research group that has led to a modification of the roadmap is the necessity for all project teams to identify and discuss, as soon as possible, the owner's intended project delivery method. This was a missing aspect of the original ID AER Roadmap and during the alignment and goal setting meeting it became apparent that this subject had a substantial impact on the level of collaboration and integration the team members engaged in, as well as on the ease with which project goals were developed. The intended project delivery contract proved to be the single most contentious factor that could compromise the gains of promoting an ID process for advanced energy retrofits. The reason being that some forms of contract delivery favor shared successes and risk amongst all team members, while others do not; those that do are more aligned with the goals of ID. As a result, an early all team discussion on the probable project delivery and procurement method to be used in completing the retrofit has now been included as part of the checklist activities within the conceptualization phase of the roadmap.

The second conclusion made by the research group is the importance of having key decision-makers involved in the process from the very beginning. As project teams discussed the range of constraints which the project would confront during each of the facilitated workshops, the need for the participation of financial and contracting decision-makers, as well as project scope decision-makers on the project team became very evident. For public entities, this is all the more important as these organizations typically have complicated procurement procedures and owner group structures with decision-makers representing a broad spectrum of departments, often with different goals and motivations. In order to truly align a project team around integrated and shared goals, the key decision-makers need to participate as soon as possible within the process, both to build will and to demonstrate the value of an integrated approach. This observation is what led to the expansion of the definition of owner group within the roadmap, as well as enhanced set of checklist activities and guidelines to identify project team roles and organizational charts within the conceptualization phase of an ID AER.

A third conclusion that can be drawn from having completed the first set of CM workshops is the importance of personal motivation on the part of team members. Building the will for integration and for meeting project goals on the part of the owner group and the team of professionals is imperative to the success of an ID AER project. If the project team does not arrive at the initial CM stage fully committed to the process, time and effort are lost. This was, in fact, the case in two of the four demonstration projects because the owners have not been decisive regarding the intended project delivery method. In addition, this was the main reason why Building E decided not to follow through with the ID AER project after the pre-project RG stage. This occurred when the most motivated member of the owner group, who championed Building E's effort to engage in an integrated process with CBEI, was no longer in a lead decision-making role for the municipality following the loss of an election. As a result, realignment of the owner group occurred and it was determined that an ID AER project would 
not be the best use of their limited funds, reallocating them to what was believed a more cost effective use in retro-commissioning the building.

Hence, as detailed above in these early conclusions of the process, there is much that can be learned about the theory of Integrated Design when put to the test of actual demonstration projects. Contracts, motivation and leadership are key factors in the completion of a building project, particularly in the completion of advanced energy retrofits. Notwithstanding the five ID AER Roadmap demonstration projects are still in their early phases, observations, notes and surveys from the various $\mathrm{CM}$ stages and $\mathrm{RG}$ stages confirm that owner groups and team members believe there to be much added value in committing to integrated design protocols in the completion of advanced energy retrofits.

\section{Acknowledgements}

The ID AER Roadmap project made extensive use of internal site assessment reports for each of the CBEI demonstration projects. The authors of this paper acknowledge Mark Stutman and his team of researchers for creating these reports and making them available to us. In addition, the authors of this paper acknowledge the work done by John Boecker to facilitate the ID process for the CBEI demonstration projects.

\section{References}

[1] Trubiano, F., Brennan, M., Albee, K. 2014. Advanced Energy Retrofit Designing Integrated Design Roadmaps. Proceedings of Purdue International High Performance Buildings Conference, (July, 2014).

[2] Albee, K., Trubiano, F., Brennan, M. 2014. Deployment and Testing of Integrated Design Roadmaps for Advanced Energy Retrofits. Proceedings of Purdue International High Performance Buildings Conference, (July, 2014).

[3] Center for Building Energy Innovation (CBEI). Building Testbed and Phased Retrofit Case Study: Building 101 at The Navy Yard in Philadelphia. Accessed May 30, 2014. http://issuu.com/alonabramson/docs/building_101 report_cbei

[4] Appleby, Paul, 2013. Sustainable Retrofit and Facilities Management. New York: Routledge.

[5] Burton, Simon, ed. 2001. Energy Efficient Office Refurbishment. London: James \& James.

[6] Liu G., B. Liu, W. Wang, J. Zhang, R. Athalye, D. Moser, E. Crowe, N. Bengtson, M. Effinger, L. Webster, and M. Hatten, 2011. Advanced Energy Retrofit Guide - Office Buildings. PNNL-20761, Pacific Northwest National Laboratory, Richland, WA.

[7] Rocky Mountain Institute. "Retrofit Depot: True Stories." Accessed November 7, 2012. http://www.rmi.org/retrofit_depot_get_connected_ true_retrofit_stories 